\title{
Ewing's sarcoma/primitive neuroectodermal tumor of the kidney: a case report and literature review
}

\author{
Shasha Zhang ${ }^{1,2}$, Yaying $\mathrm{Li}^{2}$, Rongpin Wang ${ }^{2}$, Bin Song ${ }^{1}$ \\ ${ }^{1}$ Department of Radiology, Sichuan University West China Hospital, Chengdu 610041, China; ${ }^{2}$ Department of Radiology, Guizhou Provincial \\ People's Hospital, Key Laboratory of Intelligent Medical Imaging Analysis and Accurate Diagnosis of Guizhou Province, Guiyang 550002, China \\ Correspondence to: Dr. Bin Song. Department of Radiology, Sichuan University West China Hospital, No.37 Guoxue Alley, Wuhou District, Chengdu \\ 610041, China. Email: songlab_radiology@163.com.
}

\begin{abstract}
Ewing sarcoma/primitive neuroectodermal tumor (ES/PNET) is a malignant bone, and softtissue tumor derived from neuroectoderm. It occurs in pediatric adolescents with the histopathological features of invasiveness. Renal involvement is extremely rare, which is limited to case reports and small case series. Most patients showed non-specific symptoms, such as abdominal pain and severe hematuria. The corresponding diagnosis was based on pathological features and immunohistochemical detection. So far, the characteristics of computed tomography (CT) have been rarely described in these cases. We report an 18-year-old man diagnosed with renal ES/PNET, who suffered from a sudden left flank pain associated with gross hematuria. The CT images showed an irregular soft tissue mass with a size of $7.3 \mathrm{~cm} \times 7.0 \mathrm{~cm} \times 9.0 \mathrm{~cm}$. The patient underwent laparoscopic nephrectomy of the left kidney. The final diagnosis of renal ES/PNET was confirmed by immunohistochemical detection and fluorescence in situ hybridization of the nephrectomy specimen. We want to point out that CT scanning is still a useful method for preliminary assessment in preoperative diagnosis.
\end{abstract}

Keywords: Ewing sarcoma (ES); primitive neuroectodermal tumor (PNET); kidney; computed tomography (CT); nephrectomy

Submitted Sep 04, 2019. Accepted for publication Sep 10, 2019.

doi: 10.21037/tau.2019.09.46

View this article at: http://dx.doi.org/10.21037/tau.2019.09.46

\section{Introduction}

Ewing sarcoma/primitive neuroectodermal tumor (ES/PNET) was initially described by Seemayer et al. in 1975 (1). A rare malignancy of the soft tissue, it originates from the neuroectoderm, fast-growing small round cell neoplasms, occurred in a substantial organ, very rare, especially in the kidney, accounting for only $1 \%$ of sarcoma (2). The tumor is highly malignant, grows rapidly, and metastases early to the lung, bone, and lymph node, resulting in poor prognosis (3). The pathogenesis of $\mathrm{ES} / \mathrm{PNET}$ is still unclear, though some literature reports may be related to chromosomal translocation by EWSR1 (4). For ES/PNET, surgical resection is the preferred approach for local control and get pathological diagnosis. Due to extensive invasion, difficult surgical resection, however, complete surgical resection can be achieved in only $24 \%$ of reported cases (5). Therefore, postoperative focal radiation therapy and chemotherapy are often implemented to prevent relapse and metastasis was the main means of treatment.

\section{Case presentation}

An 18-year-old man presented with persistent hematuria and left lower lumbar discomfort for 6 months. The routine blood examination showed mild anemia. Urine analysis was positive for red blood cells (RBC). Images revealed a large heterogeneous lobulated mass invading the mid part of the left kidney. Measuring $7.3 \mathrm{~cm} \times 7.0 \mathrm{~cm} \times 9.0 \mathrm{~cm}$. with areas of necrosis and hemorrhage, accompanied by the parenchyma invasion and expansion of the left kidney 

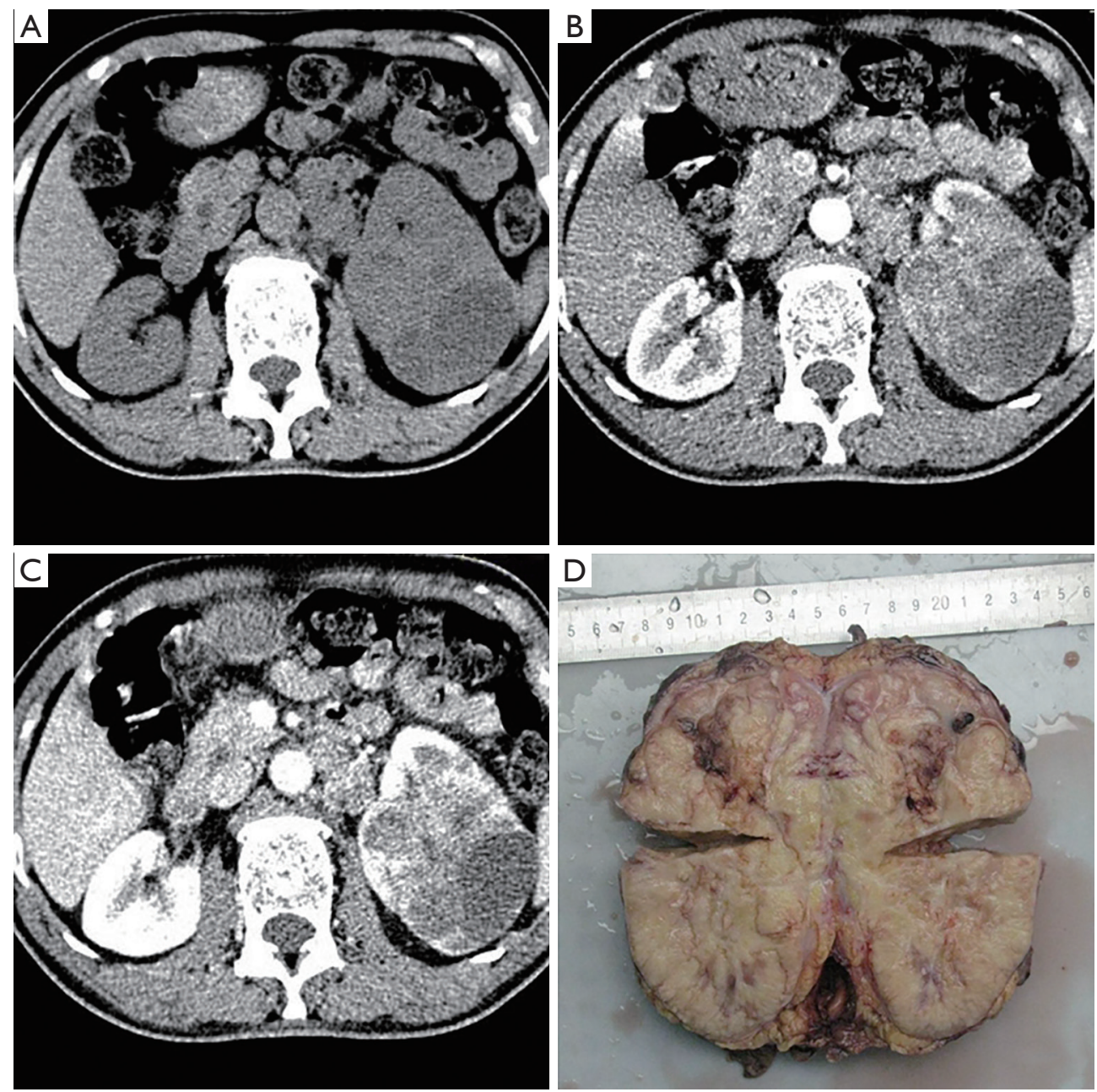

Figure $1 \mathrm{CT}$ and gross specimen images of tumor. (A) Unenhanced axial CT image shows a large multilobulated mass in the interpolar region of the left kidney; (B) corticomedullary axial CT image shows the tumor with areas of necrosis, varying degrees of irregular enhancement; (C) nephrogenic axial CT image shows a sign of tumor thrombus extends into main left kidney vein invasion; (D) gross specimen of diseased tissue. CT, computed tomography.

and sign of main renal vein invasion (Figure 1). No other abnormality was seen in the liver, pancreas, and other visceral organs. Subsequently, we performed laparoscopic nephrectomy for the patient and removed the entire left kidney successfully. Microscopically, histologic analysis of hematoxylin and eosin (H\&E) staining revealed that the tumor was composed of a monotonous population of small round cells. Extensive necrosis was also seen in histologic sections (Figure 2A). For further confirmation, immunohistochemical staining was positive for CD99, FLI-1 and SYN positive staining (Figure 2B,C,D).

Post-operatively, patients received adjuvant chemotherapy, followed by local radiotherapy. No major complication was noted during follow-up. At the most recent 12-month followup, the patient had no complaint and remained in complete remission, no sign of relapse was found.

\section{Discussion and review of the literature}

Renal ES/PNET usually presents with non-specific symptoms like those of the "classic renal tumor triads": hematuria, pain, and palpable abdominal masses. Due to its high malignancy nature, ES/PNET progresses rapidly and invade the renal collecting system early, leading to hematuria. Fewer than 150 cases were reported in the literature until now. It is a member of Ewing's sarcoma 

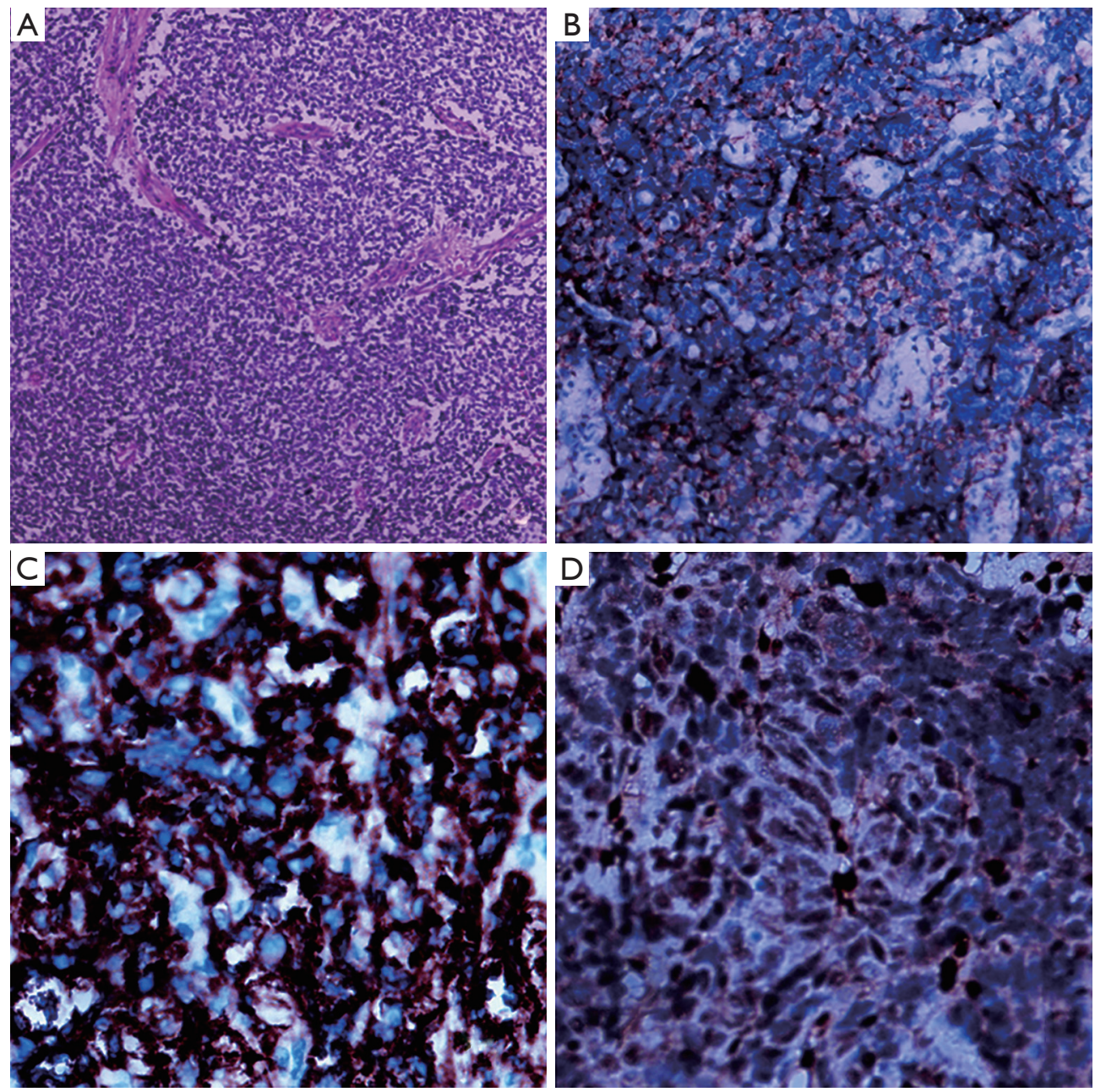

Figure 2 Histologic and immunohistochemical images of tumor. (A) Microscopically, histologic analysis of hematoxylin and eosin staining $(\times 100)$ revealed that the tumors were composed of a monotonous population of small round cells. Extensive necrosis was also observed in histologic sections; (B,C,D) immunohistochemical staining showed positive expression for CD99 ( $\times 200)$, FLI-1 ( $\times 400)$, and SYN ( $\times 400)$.

family, with aggressive clinical behavior which usually occurs in young adults most often between 20 and 30 years. The male: female ratio is about 3:1 (6). Ellinger et al. (7) reported that in one case series of 52 patients with renal ES/PNET, the average age for those affected was 26 , with $38 \%$ of all patients with the tumor between the ages of 4 and 69.

The definite diagnosis of renal ES/PNET is based on histopathology and immunohistochemistry of resected specimens. Microscopically, H\&E staining often reveals diffuse small round cells with relatively uniform size, the cytoplasm was small, the nucleus was round, the nuclear chromatin was fine, and the nucleus was not obvious, and more nuclear division can be seen. The round cells arranged in rosetted pattern is characterized in histopathology. On Immunohistochemical staining, positivity of CD99, FLI-1, and SYN are highly specific of this particular disease $(8,9)$.

Since the first description in 1994 (10), only 150 cases of renal ES/PNET have been reported with the literature. The prognosis is usually poor. ES/PNET often invaded into neighboring organs or with distant metastases, which were a high malignant tumor. According to a case series of 11 report, 8 of the follow-up time was 6 to 64 months, average 28 months; 4 cases of lungs and chest metastasis, 1 case of bone metastasis, liver metastasis, 2 cases of relapse, 5 cases of died, the average time from onset to death extensively 16.8 months (11). The largest metaanalysis including 116 cases of renal ES/PNET suggested 
a 5 -year disease-free survival rate of $45-55 \%(12,13)$. Due to its metastatic tendency, ES/PNET should receive systemic treatment once diagnosed. The recurrence rate is higher than $80 \%$ in patients who received local treatment only. Nevertheless, if no metastasis was discovered after the completion of chemotherapy, 5- and 10-year survival remarkably improved to $70 \%$ and $60 \%$, respectively (14-16).

ES/PNET of the kidney presents as a large and solitary mass of the soft tissue without a distinct boundary $(16,17)$. Computed tomography (CT) scan showed a heterogeneous mass with a low density, completely occupying the whole kidney, and its density is uneven. The high- and lowdensity areas correspond to the hemorrhage and necrosis of the specimen section, but calcification is exceedingly rare. Different degrees of enhancement can be found cystic or necrosis. We have described a case report of ES/ PNET of the kidney in a young man, which was diagnosed by immunohistochemistry analysis and FISH testing of the resected specimen. The renal ES/PNET imaging, in this case, was consistent with the above reports. The renal ES/PNET imaging, in this case, is consistent with previously reported findings $(2,7,11,16,17)$, the renal ES/PNET CT radiographical characteristics can be summarized as follows: (I) ES/PNET of the kidney affects predominantly young adults and adolescents; (II) tumors are often solitary and large; (III) on CT scan, iso-low density in the tumor. The density of the tumor is not uniform when it was large, but calcification is very rare; (IV) on contrastenhanced CT, one could see enhancement of varying degree with poorly defined margin, and, in some cases, the presence of a pseudocapsule; (V) infiltration or invasion of surrounding tissues and enlargement of lymph nodes are helpful for in making the diagnosis.

The standard treatment for ES/PNET of the kidney should be radical nephrectomy combined with postoperative radiotherapy and chemotherapy (11). Chemotherapeutic agents used were vincristine $(\mathrm{V})$, dactinomycin (D), adriamycin (A), cyclophosphamide (C), ifosfamide (I), and etoposide (E). The current standard chemotherapeutic treatment of this group involves the use of a dose-intensive combination regimen that uses these 6 drugs in a modified protocol called Ewing's family of tumors (EFT)-2001. This protocol has evolved from earlier round cell tumor II (RCT II), protocol. The main difference between the previous and current protocols is additional courses of doseintensive ifosfamide plus etoposide in the maintenance phase and weekly administration of vincristine in the induction phase in the current protocol (11). Laparoscopic nephrectomy is one of the most important techniques in nephrectomy. The first report using laparoscopic techniques nephrectomy of the kidney Ewing's sarcoma was reported by Perer et al. in 2006 (18). Compared to open surgery, Laparoscopic nephrectomy is preferred due to less morbidity and mortality (19).

\section{Conclusions}

ES/PNET of the kidney is exceedingly rare in the neuroectodermal malignant soft tissue tumor. The diagnosis of ES/PNET is based on histopathology. Because of the high invasiveness and poor prognosis of tumors, early diagnosis is crucial to intervene promptly and achieve a promising outcome. Radiologists should be aware of the key features of this malignancy on CT images during interpretation. Once diagnosed, multimodal therapy including nephrectomy, postoperative radiotherapy and chemotherapy are highly recommended to prolong survival.

\section{Acknowledgments}

None.

\section{Footnote}

Conflicts of Interest: The authors have no conflicts of interest to declare.

Ethical Statement: The authors are accountable for all aspects of the work in ensuring that questions related to the accuracy or integrity of any part of the work are appropriately investigated and resolved. Written informed consent was obtained from the patient for publication of this manuscript and any accompanying images.

\section{References}

1. Seemayer TA, Thelmo WL, Bolande RP, et al. Peripheral neuroectodermal tumors. Perspect Pediatr Pathol 1975;2:151-72.

2. Angel JR, Alfred A, Sakhuja A, et al. Ewing's sarcoma of the kidney. Int J Clin Oncol 2010;15:314-8.

3. Pakravan A, Vo TM, Sandomirsky M, et al. Primary Ewing sarcoma of kidney in an elderly. Iran J Kidney Dis 2012;6:307-10.

4. de Alava E, Pardo J. Ewing tumor: tumor biology and clinical applications. Int J Surg Pathol 2001;9:7-17. 
5. Mukhopadhyay P, Gairola M, Sharma M, et al. Primary spinal epidural extraosseous Ewing's sarcoma: report of five cases and literature review. Australas Radiol 2001;45:372-9.

6. Abolhasani M, Salarinejad S, Moslemi MK. Ewing sarcoma/primitive neuroectodermal tumor of the kidney: A report of three cases. Int J Surg Case Rep 2016;28:330-4.

7. Ellinger J, Bastian PJ, Hauser S, et al. Primitive neuroectodermal tumor: rare, highly aggressive differential diagnosis in urologic malignancies. Urology 2006;68:257-62.

8. Celli R, Cai G. Ewing Sarcoma/Primitive Neuroectodermal Tumor of the Kidney: A Rare and Lethal Entity. Arch Pathol Lab Med 2016;140:281-5.

9. Liu C, Cui LG, Wang HL. Renal Ewing's sarcoma/ primitive neuroectodermal tumor: a case report and literature review. Beijing Da Xue Xue Bao Yi Xue Ban 2017;49:919-23.

10. Mor Y, Nass D, Raviv G, et al. Malignant peripheral primitive neuroectodermal tumor (PNET) of the kidney. Med Pediatr Oncol 1994;23:437-40.

11. Thyavihally YB, Tongaonkar HB, Gupta S, et al. Primitive neuroectodermal tumor of the kidney: a single institute series of 16 patients. Urology 2008;71:292-6.

12. Risi E, Iacovelli R, Altavilla A, et al. Clinical and

Cite this article as: Zhang S, Li Y, Wang R, Song B. Ewing's sarcoma/primitive neuroectodermal tumor of the kidney: a case report and literature review. Transl Androl Urol 2019;8(5):562566. doi: $10.21037 /$ tau.2019.09.46
Pathological Features of Primary Neuroectodermal Tumor/Ewing Sarcoma of the Kidney. Urology 2013;82:382-6.

13. Bontoux C, Khaddour S, Pérot G, et al. Case report of an ewing's sarcoma/primitive neuroectodermal tumor of the kidney. Int Urol Nephrol 2018;50:1449-51.

14. Paulussen M, Ahrens S, Dunst J, et al. Localized Ewing tumor of bone: final results of the cooperative Ewing's Sarcoma Study CESS 86. J Clin Oncol 2001;19:1818-29.

15. Obata H, Ueda T, Kawai A, et al. Clinical outcome of patients with Ewing sarcoma family of tumors of bone in Japan: the Japanese Musculoskeletal Oncology Group cooperative study. Cancer 2007;109:767-75.

16. Ekram T, Elsayes KM, Cohan RH, et al. Computed tomography and magnetic resonance features of renal ewing sarcoma. Acta Radiologica 2008;49:1085-90.

17. Parada D, Godoy A, Liuzzi F, et al. Primary Ewing's sarcoma/primitive neuroectodermal tumor of the kidney. An infrequent finding. Arch Esp Urol 2007;60:321-5.

18. Perer E, Shanberg AM, Matsunaga G, et al. Laparoscopic removal of extraosseous Ewing's sarcoma of the kidney in a pediatric patient. J Laparoendosc Adv Surg Tech A 2006;16:74-6.

19. Doroudinia A, Ahmadi S, Mehrian P, et al. Primary Ewing sarcoma of the kidney. BMJ Case Rep 2019. doi: 10.1136/ bcr-2018-227198. 